\title{
Non-iterative data-driven controller tuning with guaranteed stability: Application to direct-drive pick-and-place robot
}

\author{
Klaske van Heusden, Alireza Karimi, Dominique Bonvin, Arjen den Hamer and Maarten Steinbuch
}

\begin{abstract}
This paper illustrates the practical application of non-iterative correlation-based tuning with guaranteed stability. In this method, a sufficient condition for closed-loop stability is defined as the $H_{\infty}$-norm of a particular error function. This norm is then estimated using data from one closed-loop experiment. The method is applied to a pick-and-place robot. It is shown that the proposed constraints for stability are effective without being overly conservative. Furthermore, it is shown how the method can be used to systematically design low-order controllers.
\end{abstract}

\section{INTRODUCTION}

In model reference control, the control specifications are formulated as a reference model, and the objective is to design a controller for which the controlled system resembles this reference model. A model-based solution to this problem requires the identification of a plant model, which is then used to compute the controller that minimizes the error between the reference model and the closed-loop system. This approach thus uses two optimization steps. An additional controller-order reduction step might be necessary before implementation.

Several data-driven techniques have been proposed as an alternative to model-based approaches [1], [2], [3], [4]. In these data-driven approaches, the control criterion is minimized directly with respect to the controller parameters. Compared to a model-based approach, the optimization in the identification step is omitted, and undermodeling of the plant is avoided. Furthermore, the controller structure does not depend on the structure of the model and the order of the controller can be fixed. However, closed-loop stability can in general not be guaranteed [5].

A non-iterative data-driven controller tuning approach that guarantees closed-loop stability is proposed in [6]. A sufficient condition for closed-loop stability, defined as the $H_{\infty}$-norm of some error function, is added to the model reference problem. In a data-driven setting, this condition can be verified using an estimate of the $H_{\infty}$-norm. If a spectral estimate is used, this leads to a set of constraints that is convex for a linearly parameterized controller. The resulting optimization can be solved for large data sets. The method uses the correlation approach to deal with measurement noise and is applicable to stable and unstable systems.

K. van Heusden, A. Karimi and D. Bonvin are with the Laboratoire d'Automatique of Ecole Polytechnique Fédérale de Lausanne (EPFL), 1015 Lausanne, Switzerland.

A.J. den Hamer and M. Steinbuch are with the Control Systems Technology Group, Eindhoven University of Technology, The Netherlands.

This research work is financially supported by the Swiss National Science Foundation under Grant No. 200020-105139.

Corresponding author: alireza.karimi@epfl.ch
The optimal controller parameters, calculated using the approach given in [6], satisfy the stability condition and minimize the approximate model-reference criterion, for a given reference model and controller structure. The designed controller achieves good performance if both the reference model and the structure of the controller are appropriate for the plant. In practice, the reference model and the controller structure are defined by the user, and it is not straightforward how to choose either of them.

This paper presents the results of application of the approach proposed in [6] to a pick-and-place robot. It is shown how the approach can be used to systematically design low-order controllers, starting with the design of a highorder FIR controller. An orthogonal basis is then chosen to approximate the high-order FIR controller by a controller that can actually be implemented. If the order of the controller needs to be reduced further, the main characteristics of the high-order controllers can be used to define an appropriate structure for the low-order controller. An iterative procedure is used to define the reference model, based on the windsurfing approach for iterative control design [7], where the required performance is increased gradually by increasing the bandwidth of the reference model.

The approach given in [6] is summarized in Section II. The robot is described in Section III. Controller design is discussed in Section IV, where the performed experiments are described in Section IV-A, the design of high-order controllers is treated in Section IV-B and the design of loworder controllers using the characteristics of the high-order controllers is discussed in Section IV-C. Conclusions are provided in Section V.

\section{Data-Driven Model Reference Control With GuaranteEd STABILITY}

\section{A. Model reference control with guaranteed stability}

Consider the unknown linear SISO plant $G\left(q^{-1}\right)$, where $q^{-1}$ denotes the backward shift operator. Specifications for the controlled plant are given as a stable strictly proper reference model $M\left(q^{-1}\right)$. The objective is to design a linear, fixed-order controller $K\left(q^{-1}, \rho\right)$, with parameters $\rho$, for which the controlled plant resembles the reference model $M\left(q^{-1}\right)$. This can be achieved by minimizing the twonorm of the difference between the reference model and the achieved closed-loop system:

$$
J_{m r}(\rho)=\left\|F\left[M-\frac{K(\rho) G}{1+K(\rho) G}\right]\right\|_{2}^{2} .
$$




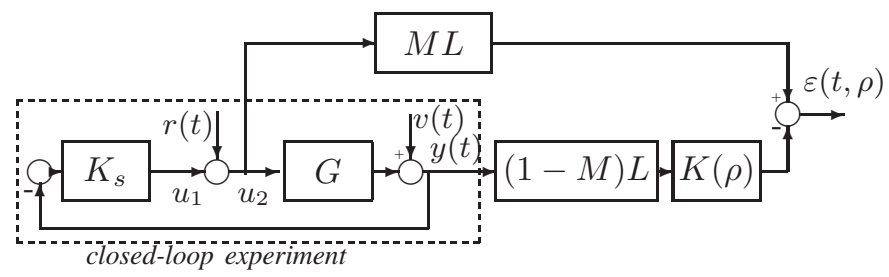

Fig. 1. Tuning scheme for model reference control problem using one closed-loop experiment

The model reference criterion (1) is non-convex with respect to the controller parameters $\rho$. An approximation that is convex for linearly parameterized controllers can be defined using the reference model $M$, see [6] for details. The approximate model-reference criterion is given by:

$$
J(\rho)=\|F(1-M)[M-K(\rho)(1-M) G]\|_{2}^{2},
$$

with $F$ a weighting filter. Note that the objective is to design a fixed-order controller and that $J(\rho)=0$ can in general not be achieved.

There is no guarantee that a controller determined by minimizing $J(\rho)$ actually stabilizes the plant. In [6], a sufficient condition for stability is added to the approximate model reference problem. The approach for closed-loop experiments is summarized next. Assume that a set of measurements from the plant controlled by the stabilizing controller $K_{s}$ is available. Note that this controller is not necessarily known, only the data are assumed to be available. The closed-loop plant for this controller is given by $M_{s}=\frac{K_{s} G}{1+K_{s} G}$. The stable controller $K(\rho)$ then stabilizes the plant $G$, if $\left.\exists \delta_{N} \in\right] 0,1[$ such that

$$
\delta(\rho):=\left\|M_{s}-K(\rho)\left(1-M_{s}\right) G\right\|_{\infty} \leqslant \delta_{N} .
$$

This stability condition is based on the small-gain theorem. The proof is given in [6], where the constraint is fixed as $\delta(\rho)<1$. In a data-driven approach, $\delta(\rho)$ is estimated and smaller values of $\delta_{N}$ can be used to compensate for the estimation error. The result stated above considers stable controllers, but special cases of unstable controllers can easily be handled, for example integrators, see [8] for details in the context of controller validation. A stabilizing solution to the approximate model reference problem is then given by:

$$
\begin{aligned}
& \rho_{s}=\arg \min _{\rho} J(\rho) \\
& \text { subject to } \delta(\rho) \leqslant \delta_{N} .
\end{aligned}
$$

\section{B. Tuning scheme}

Consider the tuning scheme shown in Fig. 1, where the plant is controlled by the stabilizing $K_{s}$ (which might be unknown). The excitation signal is applied directly to the input of the plant. The error

$$
\varepsilon(t, \rho)=L\left(M u_{2}(t)-K(\rho)(1-M) y(t)\right)
$$

can be used to compute the optimal controller. Note that the noise affects the input to the controller to be identified and not the output as in standard identification problems. The correlation approach will be used to deal with the effect of noise. A second error signal $\varepsilon_{s}(t, \rho)$, which will be used in the stability constraint, is defined as:

$$
\begin{aligned}
& \varepsilon_{s}(t, \rho)=-u_{1}(t)-K(\rho) y(t) \\
= & \left(M_{s}-K(\rho)\left(1-M_{s}\right) G\right) r(t)+\left(K_{s}-K(\rho)\right)\left(1-M_{s}\right) v(t) .
\end{aligned}
$$

The transfer function between $r(t)$ and $\varepsilon_{s}(t, \rho)$ is equal to the transfer function defining $\delta(\rho)$ in (3). The signals available from the scheme of Fig. 1 can thus be used to estimate $\delta(\rho)$.

\section{Implementation using the correlation approach}

The ideal controller $K\left(\rho^{*}\right)$ is defined as the controller that achieves $M=K\left(\rho^{*}\right) G(1-M)$. The error signal (5) corresponding to this ideal controller becomes filtered noise. Since $v(t)$ is not correlated with the reference $r(t)$, the ideal error $\varepsilon\left(t, \rho^{*}\right)$ will not be correlated with $r(t)$ either. The objective of correlation based tuning is therefore to tune the controller parameters $\rho$ such that $\varepsilon(t, \rho)$ and $r(t)$ become uncorrelated. Assume that the reference signal $r(t)$ is periodic with period $N_{p}$ and that it includes an integer number of periods $n_{p}$, i.e. the total signal length is $N=n_{p} N_{p}$. The auto-correlation of $r(t)$ is given by $R_{r}(\tau)=\frac{1}{N_{p}} \sum_{t=1}^{N_{p}} r(t-\tau) r(t)$. Assume that the spectrum of $r(t)$ satisfies

$$
\Phi_{r}\left(\omega_{k}\right)=\sum_{\tau=0}^{N_{p}-1} R_{r}(\tau) e^{-j \tau \omega_{k}} \neq 0,
$$

for $\omega_{k}=2 \pi k / N_{p}, k=0, \ldots, N_{p}-1$, and that the filter $L(1-M) G /\left(1+K_{s} G\right)$ has no zero on the imaginary axis. Furthermore, assume that the noise $v(t)$ can be represented as filtered white noise, $v(t)=H\left(q^{-1}\right) e(t)$, where $H\left(q^{-1}\right)$ is stable and assume that $v(t)$ is not correlated with the reference signal $r(t)$, i.e.

$$
R_{r v}(\tau)=\lim _{N \rightarrow \infty} \frac{1}{N} \sum_{t=1}^{N} r(t-\tau) v(t)=0 .
$$

Let the controller be linearly parametrized,

$$
K\left(q^{-1}, \rho\right)=\beta^{T}\left(q^{-1}\right) \rho, \rho \in \mathcal{D}_{K},
$$

where the set $\mathcal{D}_{K}$ is compact and $\beta\left(q^{-1}\right)$ is a vector of stable linear discrete-time transfer operators, $\beta\left(q^{-1}\right)=$ $\left[\beta_{1}\left(q^{-1}\right), \ldots, \beta_{n_{\rho}}\left(q^{-1}\right)\right]^{T} \cdot n_{\rho}$ is the number of controller parameters. The vector of instrumental variables $\zeta(t)$, correlated with $r(t)$ and uncorrelated with $v(t)$, is defined as:

$$
\zeta(t)=\left[r\left(t+l_{1}\right), \ldots r(t), \ldots, r\left(t-l_{1}\right)\right]^{T},
$$

where $l_{1}$ is a sufficiently large integer. The choice of $l_{1}$ provides a trade-off between bias and variance in the controller parameters. The correlation function is defined as

$$
f_{N, l_{1}}(\rho)=\frac{1}{N} \sum_{t=1}^{N} \zeta(t) \varepsilon(t, \rho)
$$

and the correlation criterion $J_{N, l_{1}}(\rho)$ as

$$
J_{N, l_{1}}(\rho)=f_{N, l_{1}}^{T}(\rho) f_{N, l_{1}}(\rho) .
$$


The stability constraint can be implemented using a spectral estimate. Let the error signal $\varepsilon_{s}(t, \rho)$ be generated periodically, i.e. no transients are present in the response. The cross-spectrum between $\varepsilon_{s}(t, \rho)$ and $r(t)$ can be estimated:

$$
\hat{\Phi}_{r \varepsilon_{s}}\left(\omega_{k}, \rho\right)=\sum_{\tau=0}^{N_{p}-1} \hat{R}_{r \varepsilon_{s}}(\tau, \rho) e^{-j \tau \omega_{k}},
$$

where $\hat{R}_{r \varepsilon_{s}}(\tau, \rho)=\frac{1}{N} \sum_{t=1}^{N} r(t-\tau) \varepsilon_{s}(t, \rho), \quad \tau=$ $0, \ldots, N_{p}-1$. The spectral estimate

$$
\hat{\delta}(\rho)=\max _{\omega_{k}}\left|\frac{\hat{\Phi}_{r \varepsilon_{s}}\left(\omega_{k}, \rho\right)}{\Phi_{r}\left(\omega_{k}\right)}\right|
$$

can be used to define a set of convex constraints that converges to the constraint (3). For periodic signals, the optimization problem (4) can be approximated by:

$$
\begin{gathered}
\hat{\rho}=\underset{\rho}{\arg \min _{\rho}} J_{N, l_{1}}(\rho) \\
\text { subject to } \\
\left|\sum_{\tau=0}^{N_{p}-1} \hat{R}_{r \varepsilon_{s}}(\tau, \rho) e^{-j \tau \omega_{k}}\right| \leqslant \delta_{N}\left|\sum_{\tau=0}^{N_{p}-1} R_{r}(\tau) e^{-j \tau \omega_{k}}\right|, \\
\omega_{k}=2 \pi k / N_{p}, \quad k=0, \ldots,\left\lfloor\left(N_{p}-1\right) / 2\right\rfloor .
\end{gathered}
$$

If the controller is parameterized linearly, according to (6), this optimization is convex in $\rho$. Let the stable filter $L$ be defined as

$$
L\left(e^{-j \omega_{k}}\right)=\frac{F\left(e^{-j \omega_{k}}\right)\left(1-M\left(e^{-j \omega_{k}}\right)\right)}{\Phi_{r u_{2}}\left(\omega_{k}\right)} .
$$

Then, as $N, l_{1}, N_{p} \rightarrow \infty, N_{p} / N, l_{1} / N \rightarrow 0$, the estimate $\hat{\rho}$ converges to the stabilizing solution $\rho_{s}$, see [6] for the proof. $\Phi_{r u_{2}}\left(\omega_{k}\right)$, the cross-spectrum between $r(t)$ and $u_{2}(t)$, can be estimated from the available data.

Remarks: The stability condition is defined with respect to the stabilizing controller $K_{s}$, since $\delta$ represents a distance between this $K_{s}$ and the controller to be designed. Similar ideas can be found in the windsurfing approach [7] and cautious controller tuning [9]. These are iterative methods, where the difference between consecutive controllers is limited to avoid stability problems.

In the scheme of Fig. 1, the error is filtered by $L$, see (5), which is not the case in the scheme proposed in [6]. In [6], the filtering is applied to the instrumental variables. However, these two approaches are asymptotically equivalent.

\section{Double SCARA DIRECT-Drive Robot}

The approach is applied to a pick-and-place robot known as the FAMMDD, Fast and Accurate Manipulator Modules Direct Drive. This robot has been developed by Philips CFT [10]. The robot used in the experiments has no transmission, hence the name Direct Drive. The FAMM consists of two SCARAs (Selective Compliant Assembly Robot Arms), see Fig. 2 and Fig. 3. The upper arms are fixed to two concentric axes, and the end-effector is situated at the wrist. The robot is driven by four AC motors, two in the wrist and two on the main axis. Only displacements in the horizontal plane

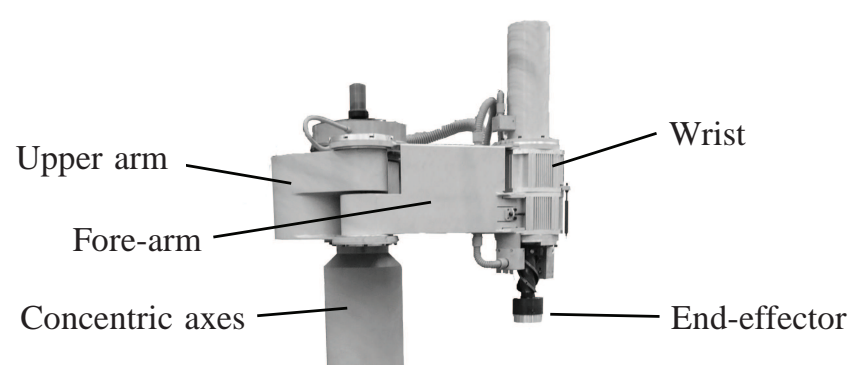

Fig. 2. FAMMDD double SCARA pick-and-place robot.

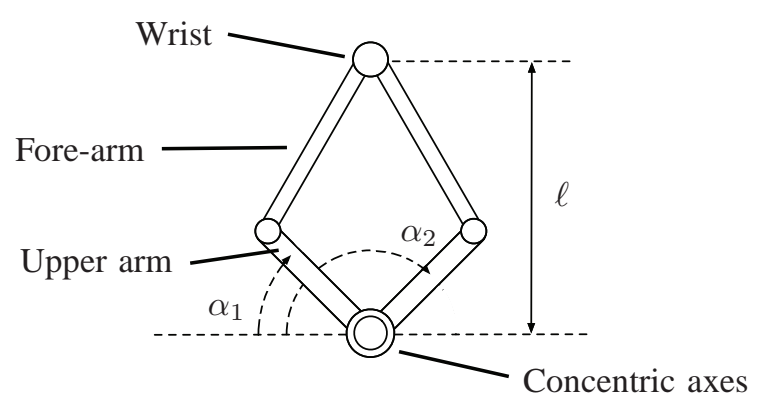

Fig. 3. Schematic representation of FAMMDD.

will be considered in the experiments, the position of the end-effector in the wrist being fixed.

Both SCARAs are driven by a servomotor integrated in the axis. Permanent magnets are fixed to the axis, which acts as the rotor of the motor. The base of the robot contains the stator coils. An advantage compared to a single SCARA robot is that the mass of the main actuators does not move as the end-effector is displaced. The arms are designed such that the moving mass is minimized, while the required stiffness is maintained. The transmission-free actuation avoids backlash and other transmission disadvantages, but the load dynamics are dominant since they are not reduced by a transmission.

The first motor drives the left arm and affects the angle $\alpha_{1}$, as defined in Fig. 3. The second motor drives the right arm affecting the angle $\alpha_{2}$. If both motors are moving in the same direction, $\alpha_{1}-\alpha_{2}=0$, the end-effector rotates around the main axis. If the motors move in opposite directions, the distance $\ell$ of the end-effector from the main axis changes. The load dynamics depend on the position $\ell$ of the endeffector, which causes nonlinear behavior.

Both angles $\alpha_{1}$ and $\alpha_{2}$ are measured. The objective is to position the end-effector, and the controlled variables are the rotation angle $\alpha=\frac{\alpha_{1}+\alpha_{2}}{2}$ and $\tilde{\ell}=\frac{\alpha_{1}-\alpha_{2}}{2}$. Note that $\ell$ is a nonlinear function of the controlled variable $\tilde{\ell}$. The implementation of this change of variables is shown in Fig. 4. The outputs of the system are $\alpha$ and $\tilde{\ell}$, the inputs are $u_{k 1}$ and $u_{k 2}$, and $u_{m 1}$ and $u_{m 2}$ are the resulting inputs to the first and second motor respectively.

If the distance of the wrist from the main axis, $\ell$, is constant, and only small rotations $\alpha$ around the axes are considered, the system is approximately linear. In the following experiments, the distance of the wrist from the main axis is 


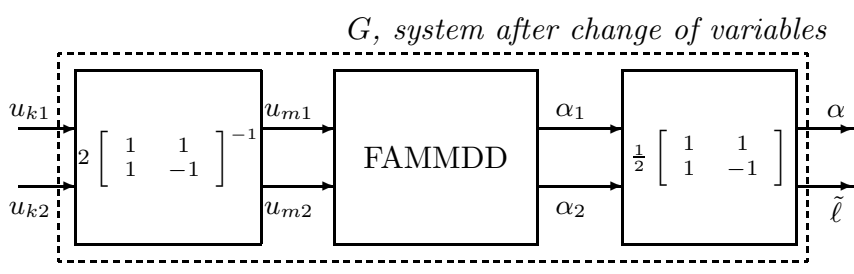

Fig. 4. Change of variables as implemented on the FAMMDD.

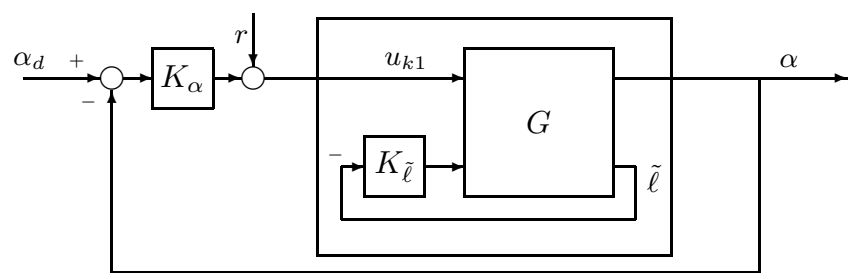

Fig. 5. Experimental scheme used for controller design.

controlled by $K_{\tilde{\ell}}$, a PD controller with a low-pass filter. The controller for the resulting SISO system with input $u_{k 1}$ and output $\alpha$ is designed using the approach of Section II.

\section{CORRELATION-BASED DATA-DRIVEN CONTROLLER DESIGN}

\section{A. Experiments}

Using the initial stabilizing controller $K_{\alpha}$, the experiment is performed in closed loop according to the scheme of Fig. 5. The system is sampled with the sampling time of $1 \mathrm{~ms}$. $r(t)$ is a PRBS with period length of $N_{p}=4095$ and amplitude 0.24 . Because there is no compensation for friction, the experiments are performed on the robot in movement. $\alpha_{d}(t)$ is a sinusoid of approximately $0.25 \mathrm{~Hz}$ with amplitude 0.6 radians, where the exact frequency is chosen such that the excitation and its harmonics due to nonlinearities are located at frequencies inbetween the frequencies $\omega_{k}$ excited by $r(t)$. The amplitude of $\alpha_{d}$ is chosen such that the number of changes in direction are limited.

A set of data of length $N=150 N_{p}$ is collected according to the scheme of Fig. 1. The DFT of these signals is used to calculate the frequency response from $u_{k 1}$ to $\alpha$, see Fig. 6 . The first anti-resonance and resonance are situated around $150 \mathrm{rad} / \mathrm{s}$.

\section{B. Design of high-order controllers}

At low frequencies, the system behaves as a double integrator. The first reference model $M_{1}$ is chosen accordingly, such that $1-M_{1}$ has two zeros at 1 :

$$
M_{1}=\frac{0.00137 q^{-4}-0.00135 q^{-5}}{A_{M}},
$$

with $A_{M}=1-3.75 q^{-1}+5.32 q^{-2}-3.42 q^{-3}+0.898 q^{-4}-$ $0.037 q^{-5}$. The bandwidth of $M_{1}$ lies below the first antiresonance of the plant and it is expected that this objective can be achieved. Due to the anti-resonances in the system, the ideal controller $K^{*}$ that achieves $M$ is expected to

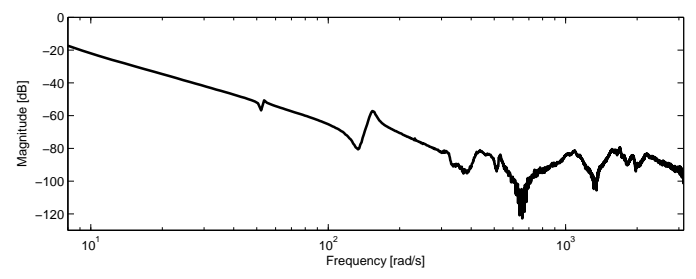

Fig. 6. Magnitude Bode diagram of measured frequency response from $u_{k 1}$ to $\alpha$.

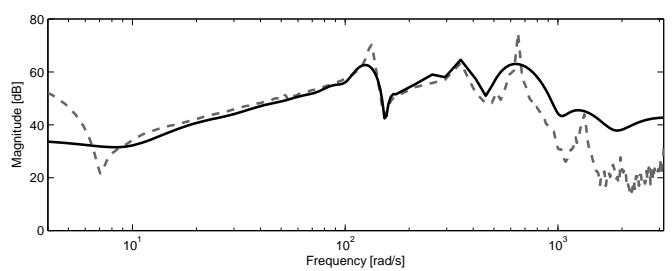

Fig. 7. Magnitude Bode diagram of calculated controllers for $M_{1}$. Grey dashed: FIR of order 1500. Black: Laguerre basis functions of order 30.

show resonant behaviour. In an FIR structure, such resonant behaviour can only be described if the order of the FIR filter is high. An FIR controller of order 1500 is designed, using the approach of Section II. The optimization is implemented using YALMIP [11] and SeDuMi [12]. Since $r(t)$ is a PRBS signal, the extended instruments of (7) can be taken as,

$$
\zeta(t)=\left[r(t), r(t-1), \ldots, r\left(t-l_{1}\right)\right]^{T} .
$$

Furthermore, the following choices are made: $F=1, l_{1}=$ $\left(N_{p}-1\right) / 2$ and $\delta_{N}=0.9$. Note that, for $N_{p}=4095$ constraints, no computational problems are encountered for the calculation of 1500 parameters.

The magnitude Bode diagram of both 1500th-order FIR controllers is shown in Fig. 7. Note that the stability constraints introduce conservatism, and the optimal controller approximates $K^{*}$ only if the constraints are not active. Inspection of the constraints shows that they are active only for very low frequencies. It is therefore assumed that, apart from low frequencies, the distance between $K^{*}$ and the high order FIR controller can be made very small, and that this controller approximates the characteristics of $K^{*}$.

The (ideal) high order controller contains two poorly damped resonances, one that cancels the first anti-resonance of the system and a second one at a higher frequency. This controller cannot be implemented, for at least two reasons. Firstly, the order of the controller is too large. Secondly, even though this controller may achieve perfect model matching for the measured output $\alpha$, it is not necessarily a good controller for the plant. For systems that contain an antiresonance, cancelation of this anti-resonance may cause oscillations in other (not necessarily measured) parts of the system.

A second controller is therefore calculated, of order 30 with an orthogonal basis of Laguerre functions with poles in 0.8 (see [13] for an overview of orthogonal basis functions in system identification). This orthogonal basis offers many de- 


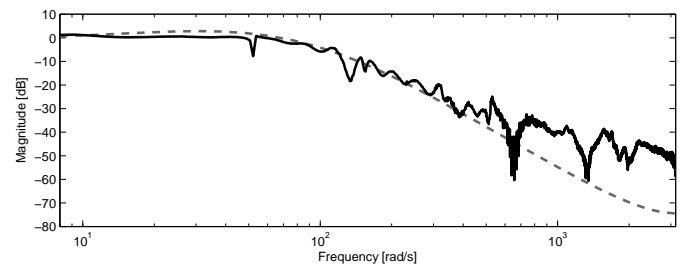

Fig. 8. Achieved closed-loop performance. Grey dashed: reference model $M_{1}$. Black: measured complementary sensitivity with 30th-order controller.

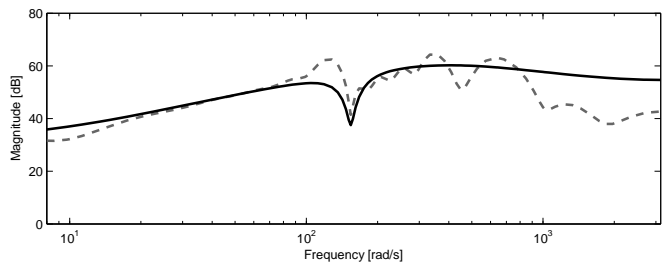

Fig. 9. Magnitude Bode diagram of calculated controllers for $M_{1}$. Grey dashed: Laguerre basis of order 30. Black: $K_{1}$ of order 4

grees of freedom at low frequencies and thus permits model matching at the frequencies that are important for closedloop performance. However, the match at high frequencies is expected to be limited. The Bode diagram of the resulting controller is shown in Fig. 7. The damping of the resonance at low frequencies is larger than the damping of the FIR controller. As expected, the controller resembles the FIR controller between 10 and $500 \mathrm{rad} / \mathrm{s}$, but the fit at higher frequencies is limited.

The 30th-order controller is implemented and the same experiment as described in Section IV-A is performed with this controller in the loop. The measured response is used to estimate the complementary sensitivity function. The achieved closed-loop performance is shown in Fig. 8. The controller structure does not permit perfect model matching, but the error is relatively small at all frequency ranges.

\section{Design of low-order controllers}

If, for practical reasons, the order of the controller needs to be reduced, the characteristics of the 1500th- and 30th-order controller can be used to choose an appropriate structure for the low-order controller. In this example, a controller of order 4 is designed, using the data measured with the 30th-order controller in the loop.

The high-order FIR controller and the 30th-order controller clearly show the behaviour of a notch filter at about $160 \mathrm{rad} / \mathrm{s}$. Some of the controller parameters of the low-order controller need to be fixed to reproduce this behaviour. The fixed part of the controller therefore includes a notch filter, designed using the response of the 30th-order controller. The remaining two poles are fixed at 0.7 . Note that it is not necessary to actually implement the 30th-order controller to design the low-order controller. The structure of the controller is given by:

$K_{1}(\rho)=\frac{\left(\rho_{0}+\rho_{1} q^{-1}+\rho_{2} q^{-1}\right)\left(1-z_{1} q^{-1}\right)\left(1-z_{2} q^{-1}\right)}{\left(1-p_{1} q^{-1}\right)\left(1-p_{2} q^{-1}\right)\left(1-p_{3} q^{-1}\right)\left(1-p_{4} q^{-1}\right)}$,

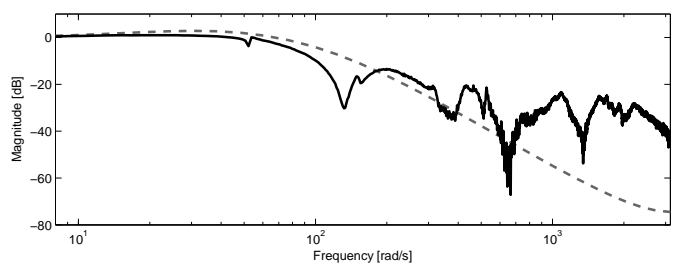

Fig. 10. Achieved closed-loop performance. Grey dashed: reference model $M_{1}$. Black: measured complementary sensitivity with $K_{1}$.

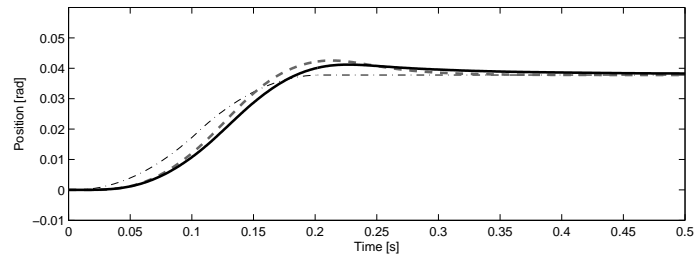

Fig. 11. Tracking performance. Dash-dot thin line: reference signal $\alpha_{d}$. Black solid: measured response with $K_{1}$. Grey dashed: response of $M_{1}$ to $\alpha_{d}$.

where $z_{1}=z_{2}^{*}=0.98+0.15 i, p_{1}=p_{2}^{*}=0.95+0.14 i$ and $p_{3}=p_{4}=0.7 . \zeta(t)$ is defined as in (14), with $l_{1}=500$, $\delta_{N}=0.9$ and $F=1$. The Bode diagram of the resulting controller is shown in Fig. 9, where the Bode diagram of the 30th-order controller is given for comparison. Inspection of the stability constraints shows that they are active for low frequencies, and the gain of the controller is limited at low frequencies to guarantee stability.

The controller is implemented and the same experiment as described in Section IV-A is performed. The controller stabilizes the plant. The achieved closed-loop performance is shown in Fig. 10. The controlled system resembles $M_{1}$ at low frequencies. At higher frequencies, the model cannot be matched due to the limited structure of $K_{1}$. However, since the controlled system resembles the reference model up to the bandwidth, the tracking performance achieved with this low-order controller is expected to be good. The time response of the controlled system is shown in Fig. 11. Note that the time responses presented in this paper are normalized for comparison. The controlled system is slightly slower than the reference model due to the stability constraints. The price to pay for stability is thus a limitation of performance.

The performance requirements can be increased by increasing the bandwidth of the reference model. A second reference model is defined as

$$
M_{2}=\frac{0.03211 q^{-4}-0.03117 q^{-5}}{A_{M_{2}}},
$$

where $A_{M_{2}}=1-3.01 q^{-1}+3.36 q^{-2}-1.68 q^{-3}+0.34 q^{-4}$ $0.013 q^{-5}$. The set of data of length $N=150 N_{p}$ collected with $K_{1}$ in the loop is used for controller design. The structure of $K_{2}(\rho)$ is the same as the structure of $K_{1}(\rho)$, $\zeta(t)$ is defined as in (14), $l_{1}=500, \delta_{N}=0.9$ and $F=1$.

For comparison, another controller $K_{l s}$ is designed using loopshaping. The non-parametric model of Fig. 6 is used to design the controller, and the cross-over frequency is 


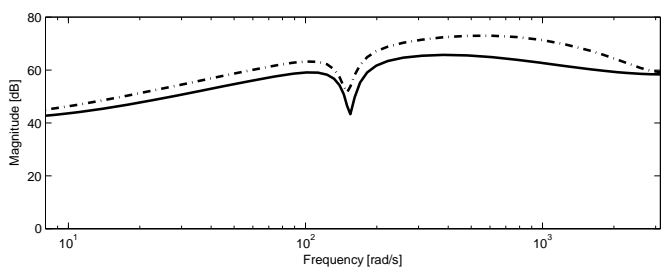

Fig. 12. Magnitude Bode diagram of calculated controllers for $M_{2}$. Black: 4th-order controller $K_{2}$. Dash-dot: 5th-order controller $K_{l s}$.

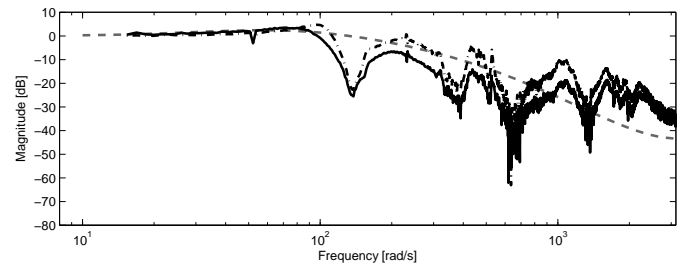

Fig. 13. Achieved closed-loop performance. Grey dashed: reference model $M_{2}$. Black: measured complementary sensitivity with $K_{2}$. Black dash-dot: measured complementary sensitivity with $K_{l s}$.

chosen similar to that of $M /(1-M)$. A notch filter is introduced to deal with the resonance. This filter is designed using the non-parametric model and is not the same as the fixed part of $K_{1}$ and $K_{2}$. A lead filter is added for the phase margin. A second-order low-pass filter is added to limit the high-frequency gain, resulting in a 5th-order controller. The Bode diagram of $K_{2}$ and of $K_{l s}$ are shown in Fig. 12. The achieved closed-loop performance is shown in Fig. 13. Model-matching up to the bandwidth is not possible with the limited controller structure. Since the control objective for $K_{2}$ is model matching, it is expected that the achieved model-reference criterion $J_{m r}$ of (1) is smaller for $K_{2}$ than for $K_{l s} . J_{m r}$ can be approximated by $\hat{J}_{m r}(K)=\sum_{\omega_{k}}\left[M_{2}\left(e^{-j \omega_{k}}\right)-T\left(e^{-j \omega_{k}}\right)\right]^{2}$, where $T\left(e^{-j \omega_{k}}\right)$ is the measured frequency response function as shown in Fig. 13. As expected, $\hat{J}_{m r}\left(K_{2}\right)<\hat{J}_{m r}\left(K_{l s}\right)$. The difference is about $3.4 \%$.

The time-domain response of the plant controlled by $K_{2}$ is comparable to that of the plant controlled by $K_{l s}$, as shown in Fig. 14. Note that the measured responses and the response of the reference model overlap. Note also that the reference signal $\alpha_{d}$ in Fig. 14 is the same as $\alpha_{d}$ in Fig. 11. $\alpha_{d}$ is not visible in the figure because the response of $M_{2}$ is much faster than the response of $M_{1}$ and the response of the reference model is superposed on $\alpha_{d}$.

The achieved tracking performance of $K_{2}$ and $K_{l s}$ are thus comparable. The Bode diagram of $K_{2}$ and that of $K_{l s}$ are also very similar. This result might not be surprising for such low-order controllers. However, it should be noted that the structure of $K_{2}$ is found systematically from a series of optimization problems, and the proposed approach can be used to calculate the optimal controller for any predefined controller structure of any order. If a higher-order controller can be implemented, the achieved performance will be improved, as illustrated by the results achieved with

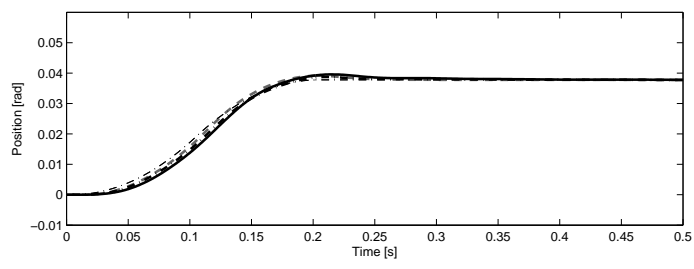

Fig. 14. Tracking performance. Dash-dot thin line: reference signal $\alpha_{d}$ Black: measured response with $K_{2}$. Black dash-dot: measured response with $K_{l s}$. Grey dashed: response of $M_{2}$ to $\alpha_{d}$.

the 30th-order controller, see Fig. 8.

\section{CONCLUSIONS}

Non-iterative correlation-based controller tuning with guaranteed stability is applied to a pick-and-place robot. A closed-loop scheme is used to generate the signals necessary for controller design. The constraints for closed-loop stability integrated in the controller design method are effective. The designed controllers stabilize the system. Low-order controllers are designed systematically. Firstly, a high-order controller is designed that represents the ideal controller. The structure of the low-order controllers is then defined such that the important characteristics of the ideal controller are maintained.

\section{REFERENCES}

[1] R. L. Kosut, "Uncertainty model unfalsification for robust adaptive control," Annual Reviews in Control, vol. 25, pp. 65-76, 2001.

[2] M. C. Campi, A. Lecchini, and S. M. Savaresi, "Virtual reference feedback tuning: A direct method for the design of feedback controllers," Automatica, vol. 38, pp. 1337-1346, 2002.

[3] H. Hjalmarsson, "Iterative feedback tuning - an overview," International Journal of Adaptive Control and Signal Processing, vol. 16, pp. 373-395, 2002.

[4] A. Karimi, L. Mišković, and D. Bonvin, "Iterative correlation-based controller tuning," International Journal of Adaptive Control and Signal Processing, vol. 18, no. 8, pp. 645-664, 2004.

[5] B. D. O. Anderson and A. Dehghani, "Challenges of adaptive controlpast, permanent and future," Annual Reviews in Control, vol. 32, no. 2, pp. $123-135,2008$.

[6] K. van Heusden, A. Karimi, and D. Bonvin, "Data-driven controller tuning with integrated stability constraint," in 47th IEEE Conference on Decision and Control, Cancun, Mexico, 2008, pp. 2612 - 2617.

[7] B. D. O. Anderson, "Windsurfing approach to iterative control design," in Iterative Identification and Control: Advances in Theory and Applications, P. Albertos and A. Sala, Eds. Berlin: Springer-Verlag, 2002.

[8] K. van Heusden, A. Karimi, and D. Bonvin, "Data-driven controller validation," in Proceedings of the 15th IFAC Symposium on System Identification, Saint-Malo, France, July 2009, pp. 1050-1055.

[9] R. R. Bitmead, "Iterative optimal control design," in Iterative Identifcation and Control: Advances in Theory and Applications, , P. Albertos and A. Sala, Eds. Berlin: Springer-Verlag, 2002.

[10] H. M. J. R. Soemers, "The design of high performance manipulators," in IEEE/ASME International Conference on Advanced Intelligent Mechatronics, 2001, vol. 1, 2001, pp. 26-31 vol.1.

[11] J. Löfberg, "YALMIP: A toolbox for modeling and optimization in MATLAB," in CACSD Conference, 2004. [Online]. Available: http://control.ee.ethz.ch/ joloef/yalmip.php

[12] J. F. Sturm, "Using SeDuMi 1.02, a Matlab toolbox for optimization over symmetric cones," Optimization Methods and Software, vol. 11, pp. 625-653, 1999.

[13] P. Heuberger, P. Van den Hof, and B. Wahlberg, Modelling and identification with rational orthogonal basis functions. London, UK: Springer-Verlag, 2005. 